\title{
Illness Perceptions Predict Cognitive Performance Validity
}

\author{
George K. Henry, ${ }^{1}$ Robert L. Heilbronner, ${ }^{2,3}$ Julie Suhr, ${ }^{4}$ Jeffrey Gornbein, ${ }^{1}$ Eveleigh Wagner, ${ }^{5}$ AND Daniel L. Drane ${ }^{6}$ \\ ${ }^{1}$ David Geffen School of Medicine at UCLA, Los Angeles, California \\ ${ }^{2}$ Chicago Neuropsychology Group, Chicago, Illinois \\ ${ }^{3}$ Feinberg School of Medicine at Northwestern University, Chicago, Illinois \\ ${ }^{4}$ Ohio University, Athens, Ohio \\ ${ }^{5}$ Vanderbilt University Medical Center, Nashville, Tennessee \\ ${ }^{6}$ Emory University School of Medicine, Atlanta, Georgia
}

(Received August 9, 2017; Final Revision February 19, 2018; Accepted February 20, 2018; First Published Online April 29, 2018)

\begin{abstract}
Objectives: The aim of this study was to investigate the relationship of psychological variables to cognitive performance validity test (PVT) results in mixed forensic and nonforensic clinical samples. Methods: Participants included 183 adults who underwent comprehensive neuropsychological examination. Criterion groups were formed, that is, Credible Group or Noncredible Group, based upon their performance on the Word Memory Test and other stand-alone and embedded PVT measures. Results: Multivariate logistic regression analysis identified three significant predictors of cognitive performance validity. These included two psychological constructs, for example, Cogniphobia (perception that cognitive effort will exacerbate neurological symptoms), and Symptom Identity (perception that current symptoms are the result of illness or injury), and one contextual factor (forensic). While there was no interaction between these factors, elevated scores were most often observed in the forensic sample, suggesting that these independently contributing intrinsic psychological factors are more likely to occur in a forensic environment. Conclusions: Illness perceptions were significant predictors of cognitive performance validity particularly when they reached very elevated levels. Extreme elevations were more common among participants in the forensic sample, and potential reasons for this pattern are explored. (JINS, 2018, 24, 735-745)
\end{abstract}

Keywords: Performance validity, Symptom validity, Illness perceptions, Health beliefs, Cogniphobia, Symptom identity

\section{INTRODUCTION}

Evaluating the validity of the psychometric database is critical in neuropsychological assessment and typically relies upon results of both performance validity testing (PVT) and symptom validity testing (SVT). PVT uses both stand alone and embedded measures such as the Word Memory Test (WMT; Green, 2005) and Reliable Digit Span (RDS; Greiffenstein, Baker, \& Gola, 1994). The validity of examinee self-report (symptom validity) is typically assessed via self-report measures such as the Minnesota Multiphasic Personality Inventory-2nd Edition-Restructured Form validity scales (MMPI-2-RF; Ben-Porath \& Tellegen, 2008). The relationship between PVT and SVT is modest at best (Haggerty, Frazier, Busch, \& Naugle, 2007), with some studies showing self-report measures, such as the MMPI-2-RF, to explain much of the variance in PVT performance (Martin, Schroeder, Heinrichs, \& Baade, 2015; Peck et al., 2013),

Correspondence and reprint requests to: George K. Henry, 11601 Wilshire Blvd., $5^{\text {th }}$ Floor, Los Angeles, California, 90025. E-mail: GHenry0249@aol.com whereas others have not (Van Dyke, Millis, Axelrod, \& Hanks, 2013).

The relationship between PVT and SVT may vary by context. In general, studies of personal injury litigants and disability claimants with external financial incentives who fail PVT (Gervais, Wygant, Sellbom, \& Ben-Porath, 2011; Nguyen, Green, \& Barr, 2015; Schroeder et al., 2012; Tarescavage, Wygant, Gervais, \& Ben-Porath, 2012; Youngjohn, Wershba, Stevenson, Sturgeon, \& Thomas, 2011; Wygant et al., 2009) show a relationship between PVT performance and over-reporting of somatic and cognitive symptoms as measured by the MMPI-2-RF Response Bias Scale (RBS; Gervais, Ben-Porath, Wygant, \& Green, 2007) and the Symptom Validity Scale (FBS-r).

In contrast, criminal defendants who fail PVT (Wygant et al., 2010; Sellbom, Toomey, Wygant, Kucharski, \& Duncan, 2010) tend to over-report psychiatric symptoms, as indicated by the Infrequent Responses Scale (F-r) and Infrequent Psychopathology Responses Scale (F-p). A recent meta-analysis of the MMPI-2-RF over-reporting scales showed large effect sizes for detecting noncredible neurocognitive dysfunction (Ingram \& Ternes, 2016). A more 
recent empirically derived embedded measure of symptom over-reporting developed for the MMPI-2-RF, the 11-item Henry-Heilbronner Index-r (HHI-r; Henry, Heilbronner, Algina, \& Kaya, 2013), has also been shown to identify symptom exaggeration in personal injury litigants and disability claimants.

Adequate performance within one validity domain does not automatically ensure adequate performance in the other (Lees-Haley, Iverson, Lange, Fox, \& Allen, 2002; Sweet, Condit, \& Nelson, 2008; Van Dyke et al., 2013). Individuals who pass both PVT and SVT are classified as producing valid test results, while individuals who fail both may be described as producing invalid test results. Test results may also be of mixed validity where subjects may fail PVT, but pass SVT, and vice versa. Under these hybrid scenarios a portion of the test results may be considered valid and the other portion invalid (Van Dyke et al., 2013). While base rates for performance invalidity vary from $30 \%$ to $54 \%$ across forensic contexts (Ardolf, Denney, \& Houston, 2007; Larrabee, 2003; Mittenberg, Patton, Canyock, \& Condit, 2002), performance invalidity ranging from $11 \%$ to $48 \%$ is also seen in nonforensic or clinical contexts with no known external incentives (An, Zakanis, \& Joordens, 2012; DeRight \& Jorgensen, 2015; Forbey \& Lee, 2011; Forbey, Lee, Ben-Porath, Arbisi, \& Gartland, 2013; Kemp et al., 2009; Schroeder \& Marshall, 2011; Silk-Eglit, et al., 2014).

There may be multiple reasons for performance invalidity. Historically, PVT failures in a forensic context with external incentives have been attributed to malingering, while failure in non-forensic contexts with no known external incentives has been claimed to be due to a variety of other factors, including mood, sleep deprivation, and fatigue (VaquezJusto, Alvarez, \& Otero, 2003); physical, emotional, or sexual abuse (Williamson, Holsman, Chayton, Miller, \& Drane, 2012); chronic pain, somatoform disorders, and medically unexplained illnesses (Drane et al., 2006; Johnson, 2008; Kemp et al., 2009; Lamberty, 2008; Suhr, 2003); interictal epileptiform activity and recent seizures (Drane et al., 2016; Loring, Lee, \& Meador, 2005); low intelligence (Dean, Victor, Boone, \& Arnold, 2008); and dementia (Dean, Victor, Boone, Philpott, \& Hess, 2009).

A survey of 316 North American neuropsychologists (Martin, Schroeder, \& Odland, 2015) revealed that the most frequent interpretation of PVT failure by examinees in a forensic context, (e.g., personal injury, disability claimants, criminal) was malingering, whereas PVT failure in a clinical non-forensic context was attributed to non-somatoform psychiatric issues. A recent selective survey of 24 board-certified North American neuropsychologists with expertise in PVT (Martin, Schroder, \& Odland, 2016) showed the majority believed that test invalidity most often resulted from malingering in a forensic context, versus somatoform/conversion disorder in non-forensic settings.

Lately, there has been increasing reticence by neuropsychologists to diagnose malingering when PVTs are failed in a forensic context. Martin and colleagues, (2015) advise, "Base rates and other relevant literature, examinee characteristics, and the nature and extent of any convergent evidence should be examined when offering explanations for validity test failure" (p. 14). In short, there is a growing interest in achieving a better understanding of both contextual and subject variables that affect performance invalidity. The present study examined several "intrinsic" or subjective psychological factors and their potential role in explaining PVT performance.

\section{Intrinsic Factors}

Self-efficacy, suggestibility, dissociation, and illness perceptions including symptom identity, illness consequences, psychological effects of illness, and cogniphobia represent just a few intrinsic psychological factors possibly influencing symptom expression and cognitive test performance. Selfefficacy is the belief, or lack thereof, that one has the capacity to perform successfully at a certain task, and to estimate how much effort will be required for successful performance. According to social cognitive theory (Bandura, 1997), individuals with high self-efficacy would be expected to have a higher probability of performance success, and vice versa. Thus, a person who believes they have an illness or injury that is responsible for their poor cognition (low self- efficacy) might not only perform more poorly on cognitive tests, but also be at greater risk of failing PVTs.

Suggestibility is a tendency to be easily influenced by what we see and hear in the world around us. According to Delis and Wetter (2007), "highly suggestible individuals may be especially prone to exaggerate cognitive dysfunction particularly in a context that reinforces a belief in those deficits" (p. 592). Highly suggestible individuals may also adopt a selective attentional bias (Mittenberg, DiGiulio, Perrin, \& Bass, 1992) causing them to "overly focus on common cognitive difficulties, interpret them as reflecting significant brain dysfunction, and possibly acting out these deficits in their daily lives or during the assessment process" (Delis \& Wetter, 2007, p. 592). Thus, high suggestibility may be associated with a greater probability of failing PVT.

Dissociation is a change in normal consciousness or awareness arising from reduced or altered access to one's thoughts feelings, perceptions, and/or memories and likely exists along a continuum. Research suggests dissociative tendencies may negatively affect basic cognitive processes including memory, attention, and executive functions (Amrhein, Hengmith, Maragkos, \& Hennig-Fast, 2008; DePrince \& Freyd, 1999; Freyd, Martello, Alvarado, Hayes, \& Christman, 1998). Thus, individuals with a greater tendency to dissociate may perform more poorly on cognitive tests and PVTs.

Symptom identity refers to the extent to which patients endorse symptoms as relevant to their current illness or injury. Thus, a person with a strong symptom identity might not only endorse a high number of symptoms, but also attribute the majority of their symptoms to a remote illness/injury. Research suggests that individuals attributing high base rate 
symptoms to a remote mild traumatic brain injury (TBI) versus other causes are more likely to report greater overall symptom severity (Belanger, Barwick, Kip, Kretzmer, \& Vanderploeg, 2013).

Illness perceptions, that is, thoughts and beliefs relative to one's diagnosis are influenced by cultural, institutional, social, and personal factors. Furthermore, individuals tend to assume their perceptions are true based upon credible information (Fragale \& Heath, 2004). Thus, symptom maintenance may be partially reinforced by well-intended healthcare providers who inform such patients their symptoms may be permanent, that is, iatrogenic effect. In contrast, reassurance, education, and discussions of expected recovery time have been shown to be effective for reducing both the magnitude and timeline of PCS complaints (Mittenberg, Tremont, Zielinski, Fichera, \& Rayls, 1996). There is some evidence that health beliefs may influence symptom reporting (Diefenbach \& Leventhal, 1996), as individuals with mild TBI who believe that their symptoms will persist and have negative consequences report significantly more postconcussive symptoms at three months (Whittaker, Kemp, \& House, 2007) and six months post-injury (Snell, Hay-Smith, Surgenor, \& Siegert, 2013).

Another factor that may influence the formation of illness perceptions is Cogniphobia (Suhr \& Spickard, 2012), a construct that arose from research documenting the effects of pain-related fear and avoidance (kinesiophobia) in health outcomes for individuals with chronic pain disorders, particularly with regard to their relationship to avoidance of physical activity (Crombez, Verbaet, Lysens, Baeyens, \& Eelen, 1998). This construct was expanded into the cognitive realm, tapping patient beliefs that engagement in mental activities demanding greater cognitive effort may actually exacerbate their neurological condition (Martelli, Zasler, Grayson, \& Liljedahl, 1999). Suhr and Spickard (2012) showed that Cogniphobia was related to diminished performance on sustained attention tasks, PVTs, and pain pressure thresholds in individuals with chronic headache. Individuals high in Cogniphobia might be expected to perform worse on PVTs.

\section{Hypotheses}

Given evidence for the potential impact of intrinsic factors on the evolution of symptom complaints and task performance, we examined the relationship of self-efficacy, suggestibility, dissociation, symptom identity, and illness perceptions to PVT performance in a mixed forensic and nonforensic clinical sample. Given the known relationship between SVT and PVT performance, we included over-reporting validity scales from the MMPI-2-RF and the HHI-r. We predicted that not only context, but also intrinsic factors (e.g., self-efficacy, suggestibility, dissociative tendency, symptom identity, and illness perceptions including consequences, psychological effects, and cogniphobia) as well as MMPI-2-RF validity scales, especially the RBS, FBS-r and HHI-r, would be predictors of PVT performance.

\section{METHODS}

\section{Background}

This multi-site 5-year study involving four neuropsychological laboratories included 198 consecutive referrals of adults ages 18-85 years, who underwent comprehensive neuropsychological examination from 2010 to 2015. The research was completed in accordance with the Helsinki Declaration. Informed consent was obtained before study inclusion. Fifteen participants were omitted due to the presence of interictal discharges during the evaluation as determined by simultaneous ambulatory electroencephalogram (EEG) recordings or video-EEG monitoring, as such activity has been shown to affect PVT performance (Drane et al., 2016; Williamson et al., 2005). The entire sample of 183 participants was comprised of private clinical patients $(n=52)$, personal injury litigants $(n=40)$, disability claimants $(n=41)$, and a mixture of university students and community members $(n=50)$ seeking neuropsychological evaluation for complaints associated with a medical condition as part of a research project. The personal injury litigants were comprised of mostly mild traumatic brain injury $(47.5 \%)$, while the most frequent diagnosis for the disability claimants was major depression (29.3\%).

\section{Performance Validity Measures}

All 183 participants were administered the Word Memory Test (WMT; Green, 2005), while a subsample of 81 examinees were also administered one additional stand-alone measure of cognitive performance validity including the Test of Memory Malingering (TOMM; Tombaugh, 1996), or the Victoria Symptom Validity Test (VSVT; Slick, Hopp, Strauss, \& Thompson, 1997). Additional embedded performance validity measures were also administered, but differed among the four laboratories based upon each laboratory's specific clinical practice and research needs. These included Reliable Digit Span (RDS; Greiffenstein et al., 1994), failure to maintain set from the Wisconsin Card Sorting Test (Greve, Heinly, Bianchini, \& Love, 2009), the Rey Auditory Verbal Learning Test (RAVLT) Delayed Recognition trial (Boone, Lu, \& Wen, 2005), Effort Index (Barash, Suhr, \& Manzel, 2004), the California Verbal Learning Test- $2^{\text {nd }}$ Edition (CVLT-2) Forced Choice Recognition (Delis, Kramer, Kaplan, \& Ober, 2000), CVLT-2 Effort Equation (Wolfe et al., 2010), and the Rey-15 Item Memory Recognition Procedure (Boone, Salazar, Lu, Warner-Chacon, \& Razani, 2002).

\section{Traditional Symptom Validity Measures}

Self-report symptom validity was assessed via the MMPI-2-RF. We included the five over-reporting validity scales of the MMPI-2-RF (F-r, Fp-r, Fs, FBS-r, RBS), as well as the HHI-r (Henry et al., 2013).

\section{Psychological Scales and Questionnaires}

All participants were administered psychological measures to assess self-efficacy, suggestibility, dissociation, symptom 
identity, and illness perceptions including consequences and cogniphobia. Self-efficacy was measured via the SelfEfficacy Scale (SES; Schwarzer \& Jerusalem, 1995), a 10-item self-report measure with scores ranging from 0 to 40 (higher scores representing greater self-efficacy). Suggestibility was examined via the 21-item Short Suggestibility Scale (SSS; Kotor, Bellman, \& Watson, 2004); scores range from 0 to 84 with higher scores representing greater suggestibility. Dissociation was evaluated via the 28 -item Dissociation Experiences Scale (DES; Bernstein \& Putnam, 1986) with scores ranging from 0 to 112 (higher scores representing greater tendency to dissociate when not under the influence of alcohol or other drugs).

Symptom Identity was quantified using an adapted version of the Illness Perception Questionnaire-Revised (IPQ-R; Moss-Morris et al., 2002) which is commonly used to assess illness perception consistent with the health beliefs model. The IPQ- $R$ is designed to be revised so that the symptomatic items are consistent with the illness/disorder of interest. Therefore, the Symptom Identity Scale, which asks participants to respond yes/no whether they have experienced a symptom since their illness/injury onset, and yes/no whether they believe the symptom is related to their illness/ injury, included 31 items likely to be associated with neuropsychological presentations. The score for the 31 (Symptom Identity Scale) items was the total number of "yes" endorsement of symptoms they believed were related to their illness/injury.

Illness perceptions were also measured using two constructs from the IPQ-R including: (1) Consequences (perception that the consequences of their illness or injury are severe) and (2) Psychological Effects (perception that their illness or injury has significant emotional and psychological effects on their day to day functioning). We added to those two constructs five additional items that assessed Cogniphobia via a Likert scale format with higher scores indicative of greater Cogniphobia (perception that cognitive effort will exacerbate neurologic symptoms). The Cogniphobia items were initially developed by Todd, Martelli, and Grayson (1998) for use in headache and were further tested by Suhr and Spickard (2012) in a headache population, where they were found to be internally consistent and to relate to other measures of pain catastrophizing and pain dangerousness, as well as worse performance on sustained attention tasks, a performance validity measure (suggesting poor effort), and lower pain pressure thresholds on the head.

\section{Word Memory Test and Gatekeeping}

Given that all 183 participants were administered the WMT, we used scores on the WMT as a "gatekeeper" to initially sort examinees into a Credible Group (CG: Pass WMT), or Noncredible Group (NCG: Fail WMT). The WMT has the added benefit of eliminating false positive classification errors by identifying examinees who produce a Genuine Memory Impairment Profile (GMIP), which is typically associated with dementia syndromes. No examinee displayed a GMIP. The Noncredible Group (NCG, $n=72$ ) was further refined by including only participants who, in addition to failing the WMT, also failed one additional stand-alone or embedded cognitive performance validity measure. The failure rates for all cognitive performance validity measures are summarized in Table 1.

The Credible Group (CG, $n=111$ ) was comprised of all subjects who initially passed the WMT and did not fail any embedded or additional stand-alone PVT. Seven subjects in the CG who passed the WMT but failed one embedded measure of performance validity were assigned an "ambiguous" credibility status and removed from the CG for any final analyses. The participants in the NCG were comprised of the following broad diagnostic categories: neurologic (54.2\%), psychiatric $(32.8 \%)$, and general medical $(13 \%)$, while participants in the CG were represented by $67.6 \%$ neurologic, $26.6 \%$ general medical, and $6.8 \%$ psychiatric patients. The two groups did not differ significantly on age $(p=.13)$, years of education $(p=.08)$, or gender $(p=.07)$, but did differ on ethnicity $(p=.01)$. Group demographics are presented in Table 2.

The composition of specific diagnoses within each diagnostic category is presented in Table 3. Participants were diagnosed with mild traumatic brain injury according to published criteria by the American Congress of Rehabilitation Medicine (1993). Epilepsy and psychogenic nonepileptic seizures were diagnosed on the basis of video-EEG

Table 1. Cognitive performance validity failure rates for the noncredible group $(n=72)$

\begin{tabular}{lc}
\hline \hline Performance Validity Test & Noncredible group \\
\hline $\begin{array}{l}\text { Word Memory Test } \\
(\leq 82.5 \% \text { IR, DR, CNS) }\end{array}$ & $72 / 183=39.3 \%$ \\
Test of Memory Malingering & \\
$(\leq 44$ Trial 2$)$ & $38 / 76=50 \%$ \\
Victoria Symptom Validity Test & \\
$(\leq 44)$ & $6 / 14=42.9 \%$ \\
$\begin{array}{l}\text { Reliable Digit Span } \\
(\leq 6)\end{array}$ & $23 / 151=15.2 \%$ \\
WCST: Failure to Maintain Set & \\
$(\geq 3)$ & $10 / 90=11.1 \%$ \\
RAVLT Delayed Recognition & \\
$\leq 10$ & $21 / 103=20.4 \%$ \\
RAVLT Effort Index & \\
$(\geq 2)$ & $8 / 49=16.3 \%$ \\
CVLT-2 Effort Equation & $10 / 49=20.4 \%$ \\
$(>.6293)$ & $1 / 21=4.8 \%$ \\
CVLT-2 LDFCR & \\
$\leq 15$ & $4 / 74=5.4 \%$ \\
Rey-15 Recognition & \\
$\leq 20$ & \\
\hline \hline
\end{tabular}

Note: Cutscores in parentheses.

$\mathrm{IR}=$ Immediate Recall, $\mathrm{DR}=$ Delayed Recall, $\mathrm{CNS}=$ Consistency of Recall; WCST $=$ Wisconsin Card Sorting Test; RAVLT $=$ Rey Auditory Verbal Learning Test; CVLT-2 LDFCR $=$ California Verbal Learning Test- $2^{\text {nd }}$ Edition Long Delay Forced Choice Recognition. 
Table 2. Demographic and participant characteristics for the credible and noncredible groups

\begin{tabular}{lcc}
\hline \hline Sample characteristics & $\begin{array}{c}\text { Credible group } \\
(n=111)\end{array}$ & $\begin{array}{c}\text { Noncredible group } \\
(n=72)\end{array}$ \\
\hline Age at testing $M(S D)$ & $43.4(18.4)$ & $47.2(15.4)$ \\
Education level $M(S D)$ & $14.7(2.7)$ & $14.2(3.0)$ \\
Gender & $37 \%$ male & $51 \%$ male \\
Race & $88.3 \%$ White & $73.6 \%$ White \\
& 6.6\% African-Am & $11.1 \%$ African-Am \\
& $4.5 \%$ Latino & $9.7 \%$ Middle Eastern \\
& $0.9 \%$ Other & $5.6 \%$ Latino \\
\hline \hline
\end{tabular}

monitoring at a tertiary care epilepsy center. Their neuropsychological evaluations were completed on the monitoring unit while undergoing video-EEG or as an outpatient with simultaneous ambulatory EEG.

\section{Data Analysis}

Data were checked for invalid response sets and outliers. No participants were removed from data analysis due to an

Table 3. Diagnostic classes and diagnoses for the credible and noncredible groups

\begin{tabular}{|c|c|c|c|}
\hline \multicolumn{2}{|c|}{$\begin{array}{l}\text { Credible group } \\
\quad(n=111)\end{array}$} & \multicolumn{2}{|c|}{$\begin{array}{l}\text { Noncredible group } \\
\qquad(n=72)\end{array}$} \\
\hline Neurologic & $n=75(67.6 \%)$ & Neurologic & $n=39(54.2 \%)$ \\
\hline Epilepsy & $n=34(45.3 \%)$ & Mild TBI & $n=23(58 \%)$ \\
\hline PNES & $n=9(12 . \%)$ & Moderate TBI & $n=4(10.2 \%)$ \\
\hline Indeterminate Szs & $n=7(9.3 \%)$ & Severe TBI & $n=3(7.7 \%)$ \\
\hline Moderate TBI & $n=7(9.3 \%)$ & Toxic exposure & $n=3(7.7 \%)$ \\
\hline Mixed Szs & $n=5(6.7 \%)$ & Stroke & $n=2(5.1 \%)$ \\
\hline Mild TBI & $n=4(5.3 \%)$ & Anoxia & $n=2(5.1 \%)$ \\
\hline Severe TBI & $n=4(5.3 \%)$ & Brain tumor & $n=1(2.6 \%)$ \\
\hline Stroke & $n=3(4.0 \%)$ & & \\
\hline Brain tumor & $n=1(0.1 \%)$ & & \\
\hline Toxic exposure & $n=1(0.1 \%)$ & & \\
\hline Psychiatric & $n=6(5.4 \%)$ & Psychiatric & $n=24(33.3 \%)$ \\
\hline Bipolar & $n=2(33.3 \%)$ & Major depression & $n=11(45.8 \%)$ \\
\hline PTSD & $n=2(33.3 \%)$ & PTSD & $n=11(45.8 \%)$ \\
\hline GAD & $n=1(16.7 \%)$ & GAD & $n=2(8.2 \%)$ \\
\hline Major depression & $n=1(16.7 \%)$ & & \\
\hline General medical & $n=30(27 \%)$ & General medical & $n=9(12.5 \%)$ \\
\hline Chronic pain & $n=10(33.3 \%)$ & Chronic pain & $n=3(33.3 \%)$ \\
\hline COPD & $n=4(13.3 \%)$ & $\begin{array}{l}\text { Obstructive } \\
\text { sleep apnea }\end{array}$ & $n=2(22.2 \%)$ \\
\hline Hypothyroidism & $n=4(13.3 \%)$ & $\begin{array}{l}\text { Chronic fatigue } \\
\text { syndrome }\end{array}$ & $n=1(11.1 \%)$ \\
\hline Diabetes & $n=4(13.3 \%)$ & $\begin{array}{l}\text { Steven-Johnson } \\
\text { syndrome }\end{array}$ & $n=1(11.1 \%)$ \\
\hline CSF & $n=3(10.0 \%)$ & Lyme disease & $n=1(11.1 \%)$ \\
\hline OSA & $n=2(6.7 \%)$ & Epstein-Barr virus & $n=1(11.1 \%)$ \\
\hline Hyperthyroidism & $n=2(6.7 \%)$ & & \\
\hline $\begin{array}{l}\text { Epstein-Barr } \\
\text { virus }\end{array}$ & $n=1(3.3 \%)$ & & \\
\hline
\end{tabular}

Note: $\mathrm{TBI}=$ traumatic brain injury; $\mathrm{PNES}=$ pseudoneurologic epileptic seizures; $\mathrm{PTSD}=$ post-traumatic stress disorder; $\mathrm{GAD}=$ generalized anxiety disorder; $\mathrm{COPD}=$ chronic obstructive pulmonary disease; $\mathrm{CSF}=$ chronic fatigue syndrome; OSA = obstructive sleep apnea; Szs = seizures .
MMPI-2-RF invalid response set (TRIN, VRIN >80T; $>15$ omissions), and there were no outliers. We first conducted a bivariate logistic regression looking at each predictor, one at a time, without controlling for the other 13 variables. Linearity between each continuous predictor and the noncredible $\log$ odds (logit) was assessed using restricted cubic splines. We followed up the bivariate logistic regression with a backward stepwise multivariate (multivariable) logistic regression by simultaneously considering all 14 potential predictor variables including 1 categorical variable (forensic) and 13 continuous predictors, including the 5 MMPI-2-RF over-reporting validity scales (F-r, Fp-r, Fs, FBS-r, RBS), the HHI-r, and 7 "intrinsic" psychological variables including self-efficacy, suggestibility, dissociation, and illness perceptions including consequences, psychological effects, Symptom Identity, and Cogniphobia.

The model was also modified to use dichotomized FBS$\mathrm{r}>17$ (equivalent to $80 \mathrm{~T}$ in the MMPI-2-RF normative sample), and Symptom Identity $>24$ in place of their continuous versions to account for nonlinearity. A $p<.05$ variable retention criterion was used. In addition a gradient boost regression (Tutz \& Binder, 2006) was also carried out using the same candidates as an alternative strategy for variable selection. Correlations and the variance inflation factors were examined among the 14 predictor variables to evaluate collinearity. Model accuracy statistics [receiver operating characteristics curve (ROC) curve area, sensitivity, specificity, and accuracy defined as the average of sensitivity and specificity] are reported. The R Foundation for Statistical Computing software, version 3.2.2, was used for most computations.

\section{RESULTS}

\section{Bivariate and Multivariate Logistic Regression}

Table 4 shows the results of the bivariate logistic regression. The odds ratio (OR) is reported for a one-unit increase in any predictor except for FBS-rRaw and Symptom Identity. For example, the $\mathrm{OR}=1.30$ for Cogniphobia suggests that the odds (not risk) of PVT failure (vs. no failure) increase 1.3 times on average for every one-unit increase in the raw Cogniphobia score.

In the bivariate logistic regression 13 of the 14 variables were significant predictors of PVT status. Only the Short Suggestibility Scale was not significant. One predictor, SelfEfficacy Scale, was associated with an OR less than 1, indicating that as scores declined the rate of PVT failure increased. All other predictors in the bivariate analysis were associated with positive ORs, indicating that as scores increased on the psychological predictors the rate of PVT failure also increased. However, 2 of the 13 continuous predictor variables, FBS-rRaw and Symptom Identity Scale, were dichotomized as they were found to have a nonlinear relation with the noncredible logit.

The multivariate logistic regression identified a final set of two psychological variables and one context variable as 
Table 4. Bivariate OR from logistic regression using one predictor at a time

\begin{tabular}{lcccc}
\hline \hline Psychological variable & OR & Lower & Upper & $p$-Value \\
\hline F-r & 1.05 & 1.03 & 1.07 & .0000 \\
Fp-r & 1.03 & 1.00 & 1.05 & .0307 \\
Fs & 1.03 & 1.01 & 1.05 & .0016 \\
FBS-rRaw $>17$ & 4.84 & 2.20 & 10.5 & .0001 \\
RBSRaw & 1.26 & 1.15 & 1.37 & .0000 \\
HHI-rRaw & 1.30 & 1.16 & 1.47 & .0000 \\
SES10 & 0.91 & 0.87 & 0.96 & 0.0001 \\
SSS21 & 1.00 & 0.97 & 1.02 & 0.7474 \\
DES28 & 1.02 & 1.00 & 1.05 & 0.0353 \\
IBQ-Illness Consequences & 1.20 & 1.08 & 1.33 & 0.0004 \\
IBQ-Psychological Effects & 1.15 & 1.06 & 1.24 & 0.0005 \\
IBQ-Cogniphobia & 1.30 & 1.18 & 1.45 & 0.0005 \\
IPQ-RN-Symptom Identity $\geq 24$ & 30.8 & 6.99 & 135.8 & 0.0006 \\
Forensic & 4.99 & 2.52 & 9.86 & 0.0001 \\
\hline \hline
\end{tabular}

Note: $\mathrm{F}-\mathrm{r}=$ Infrequent Responses Scale; Fp-r = Infrequent Psychopathology Responses Scale; Fs $=$ Infrequent Somatic Responses Scale; FBS-r $=$ Symptom Validity Scale; RBS $=$ Response Bias Scale; HHI- $r=$ HenryHeilbronner Index; SES10 = Self-Efficacy Scale; SSS21 $=$ Short Suggestibility Scale; DES = Dissociative Experiences Scale; IBQ = Illness Beliefs Questionnaire; IPQ-RN = Illness Perception Questionnaire-Revised Neurologic; $\mathrm{OR}=$ Odds ratio for one unit increase in variable; Lower and Upper $=95 \%$ confidence intervals; $\mathrm{OR}=$ odds ratio.

simultaneously significant predictors of noncredible PVT performance [area under the ROC curve $(A U C)=0.812$ ]. In decreasing order of significance, the three predictors were Cogniphobia $(\mathrm{OR}=1.21, p=.0014)$, Symptom Identity $\geq 24$ $(\mathrm{OR}=11.6 ; p=.0018)$, and forensic context $(\mathrm{OR}=2.84$; $p=.0102$ ) (see Table 5). The gradient boost regression also gave the same results.

The model posits that for every one unit increase in the raw Cogniphobia score, the odds of PVT failure increase 1.21 times, controlling for Symptom Identity and forensic status. For Symptom Identity, the OR of 11.60 suggests that examinees with a Symptom Identity score $\geq 24$ have more than an 11-fold odds of PVT failure compared to those with a Symptom Identity score $\leq 24$, that is, a "threshold" effect. The odds of PVT failure were 2.84 times greater in examines

Table 5. Multivariable Logistic Model for the Noncredible Versus Credible Group Using 3 Psychological Predictors as Candidates

\begin{tabular}{lccrr}
\hline \hline Psychological variable & OR & Lower & Upper & $p$-Value \\
\hline IBQ-Cogniphobia & 1.21 & 1.08 & 1.36 & $.0014^{*}$ \\
IPQ-RN-Symptom Identity $\geq 24$ & 11.6 & 2.48 & 54.30 & $.0018^{*}$ \\
Forensic & 2.48 & 1.28 & 6.30 & $.0102^{*}$ \\
\hline \hline
\end{tabular}

Note: $* p$-Value significant $<.01 ;$ ROC AUC $=0.812$ and is the Concordance or $\mathrm{C}$ statistic $(\mathrm{C}$ stat $=$ ROC AUC), which is a measure of accuracy; AIC $=$ 174.1 and is the Akaike Information Criterion, which is a measure of the relative quality of statistical models for a given set of data; sensitivity $=$ $60.2 \%$, specificity $=88 \%$, accuracy $=74.1 \%, \mathrm{n}=183$; OR $=$ odds ratio for one unit increase in variable; Lower and Upper $=95 \%$ confidence intervals. $\mathrm{IBQ}=$ Illness Beliefs Questionnaire; IPQ-RN = Illness Perception Questionnaire-Revised Neurologic. tested under a condition of external financial incentives (forensic context $=54.3 \%$ fail) versus non-forensic context (19.2\% fail).

Forensic participants scored significantly higher on Cogniphobia $(M=16.94 \pm 4.07)$ compared to clinical patients $(M=14.57 \pm 3.76 ; p<.001)$. A Cogniphobia raw score of $\geq 19$ was associated with $\geq .90$ specificity. Thirty-two of 172 participants (11 of the 183 missing data) scored $\geq 19$. Most high scorers $(n=24$ or $75 \%)$ were forensic, which was significantly greater than the number of high scorers who were clinical patients $(n=8 ; 25 \%)$ according to the exact binomial test. Forensic participants also scored significantly higher on Symptom Identity $(M=18.75 \pm 7.04)$ compared to clinical patients $(M=11.91 \pm 6.70 ; p<.001)$. A Symptom Identity raw score $\geq 24$ was associated with $\geq .90$ specificity. Twentyseven of 167 participants (16 of the 183 missing data) scored $\geq 24$. Most $(n=24 ; 89 \%)$ were forensic, which was significantly greater than the number of clinical patients $(n=3$; $12 \%$ ) according to the exact binomial test.

\section{Stepwise Logistic Regression by Candidate Blocks}

To further investigate the accuracy of our model, we performed a logistic regression blocking procedure to analyze whether the "intrinisic" variables are related to PVT failure once forensic context (presence/absence of external incentive), and invalid symptom report (MMPI-2-RF F-family validity scales and the HHI-r) have been controlled. First, we entered forensic context, which was a significant predictor $(p<.001)$ of PVT status $(\mathrm{AUC}=.679)$. Next, we block entered the five MMPI-2-RF symptom over-reporting scales (F-r, Fs, Fp-r, FBS-rRaw >13, RBS) as well as the HHI-r scale. Forensic context remained a significant predictor $(p<.001)$, while only the RBS was retained as a significant predictor $(p<.001)$ with an AUC $=.769$.

Finally, we block entered all 7 "intrinsic" psychological constructs (Self-Efficacy, Suggestibility, Dissociation, and Symptom Identity $>24$, Illness Perception Consequences, Illness Perception Psychological Effects, and Cogniphobia) as well as RBS and forensic context. The final model identified the same three significant predictors of PVT performance that our non-blocking, multivariate logistic regression analysis produced: forensic context $(p=.01)$, Cogniphobia $(p=.001)$, and Symptom Identity $>24(p=.002)$ with an $\mathrm{AUC}=.812$.

\section{Collinearity and Variance Inflation Factors}

The highest correlation among the three predictors in our multivariate model was $r=0.46$ (Symptom Identity and Cogniphobia). There was no evidence of collinearity. The highest correlation among all potential predictors $(r=.81)$ was between FBS-r and HHI-r, but this was not surprising as eight of the 11 HHI-r items are also found on the 30-item FBS-r. The two intrinsic predictors, that is, Cogniphobia and Symptom Identity, showed moderate correlations with RBS ( $r=.461$ and $r=.509$, respectively) and FBS-r ( $r=.455$ and 
Table 6. Correlational matrix for all 14 potential predictors of PVT performance

\begin{tabular}{|c|c|c|c|c|c|c|c|c|c|c|c|c|c|c|}
\hline & F-r & Fp-r & Fs & FBS-r & RBS & HHI-r & SES & SSS & DES & SIS & $\mathrm{CON}$ & PSY & $\mathrm{COG}$ & FOR \\
\hline F-r & 1.00 & .465 & .623 & .678 & .737 & .625 & -.532 & .115 & .528 & .508 & .283 & .447 & .470 & .250 \\
\hline Fp-r & .465 & 1.00 & .429 & .327 & .342 & .356 & -.152 & .144 & .416 & .171 & .155 & .224 & .300 & .022 \\
\hline Fs & .623 & .429 & 1.00 & .556 & .568 & .497 & -.334 & .067 & .487 & .307 & .213 & .226 & .381 & .060 \\
\hline FBS-r* & .678 & .327 & .556 & 1.00 & .748 & .812 & -.491 & .036 & .411 & .613 & .285 & .442 & .455 & .354 \\
\hline RBS* & .737 & .342 & .568 & .748 & 1.00 & .710 & -.499 & .039 & .450 & .509 & .278 & .444 & .461 & .349 \\
\hline HHI-r* & .625 & .356 & .497 & .812 & .710 & 1.00 & -.529 & .083 & .432 & .598 & .335 & .511 & .464 & .402 \\
\hline SES & -.532 & -.152 & -.334 & -.491 & -.499 & -.529 & 1.00 & .010 & -.260 & -.381 & -.162 & -.361 & -.229 & -.315 \\
\hline SSS & .115 & .144 & .067 & .036 & .039 & .083 & .010 & 1.00 & .262 & .127 & .023 & .078 & .123 & .011 \\
\hline DES & .528 & .416 & .487 & .411 & .450 & .432 & -.260 & .262 & 1.00 & .292 & .311 & .275 & .422 & .132 \\
\hline SIS & .508 & .171 & .307 & .613 & .509 & .598 & -.381 & .127 & .292 & 1.00 & .479 & .500 & .385 & .442 \\
\hline $\mathrm{CON}$ & .283 & .155 & .213 & .285 & .278 & .335 & -.162 & .023 & .311 & .479 & 1.00 & .577 & .453 & .366 \\
\hline PSY & .447 & .224 & .226 & .442 & .444 & .511 & -.361 & .078 & .275 & .500 & .577 & 1.00 & .522 & .381 \\
\hline $\mathrm{COG}$ & .470 & .300 & .381 & .455 & .461 & .464 & -.229 & .123 & .422 & .385 & .453 & .522 & 1.00 & .285 \\
\hline FOR & .250 & .022 & .060 & .354 & .349 & .402 & -.315 & .011 & .132 & .442 & .366 & .381 & .285 & 1.00 \\
\hline
\end{tabular}

Note: $*=$ Raw Score; SES = Self-Efficacy Scale; SSS = Short Suggestibility Scale; DES = Dissociative Experiences Scale; SIS = IPQ-RN Symptom Identity Scale; CON = IBQ-Consequences Scale; PSY = IBQ-Psychological Effects Scale.

$r=.613$, respectively). There were no significant interactions among the three predictors (overall $p=.218$ ). The correlational matrix is presented in Table 6 .

\section{DISCUSSION}

Results revealed that illness perceptions including Cogniphobia (perception that cognitive effort will exacerbate neurological symptoms) and Symptom Identity (perception that current symptoms are the result of illness/injury), and forensic context best predicted outcomes on PVTs in a large mixed forensic and nonforensic clinical sample.

Our prediction that MMPI-2-RF over-reporting validity scales and the HHI-r would be significant predictors of PVT failure was not supported. The failure of any of the MMPI2-RF over-reporting validity scales to emerge as significant predictors of PVT was unexpected given the bulk of clinical research supporting such a relationship (Ingram \& Ternes, 2016) showing the relative higher sensitivity of the MMPI2-RF validity scales, and especially the RBS, a scale that was developed based on MMPI-2 items that distinguished 1212 non-head-injured disability claimants who passed PVTs versus failed PVTs (Gervais et al., 2007).

Methodological differences may help to explain why RBS did not survive our analyses. A review of prior PVT studies revealed that some confined their analyses to only the RBS and other MMPI-2-RF validity scales (Jones, Ingram, \& Ben-Porath, 2012; Nguyen et al., 2015; Rogers, Gillard, Berry, \& Granacher, 2011; Wygant et al., 2009, 2010;), while others included the Substantive scales (Gervais et al., 2011; Jones et al., 2012; Nelson et al., 2011; Sellbom \& Bagby, 2010; Sellbom et al., 2010; Tarescavage et al., 2012; Thomas \& Youngjohn, 2009). The majority of studies used some version of analysis of variance, while others relied upon a regression analysis or used correlational matrices to select predictor variables. Furthermore, predictor variables of PVT performance were not simultaneously considered.
Although the RBS was a significant predictor in our bivariate logistic regression, it did not remain a significant predictor of PVT status in our multivariate logistic regression. This may have been due to the addition of our "intrinsic factors" coupled with simultaneous consideration of all predictors.

Our prediction that intrinsic factors would be related to PVT failure was partially supported. These included Cogniphobia and Symptom Identity, which were associated with $\log$ OR values greater than 1 , indicating that, as scores increased on Cogniphobia or Symptom Identity, the risk of PVT failure also increased. A Cogniphobia score of $\geq 19$ was associated with .92 specificity, .35 sensitivity, and overall classification accuracy of $65.7 \%$ for PVT failure. Thirty-two of 172 participants (18.6\%) scored $\geq 19$ on the Cogniphobia Scale. However, a within-group analysis revealed that most of the high scorers $(75 \%)$ were examined in a forensic context, which was significantly greater than the percentage of clinical nonforensic participants scoring $\geq 19$ on the Cogniphobia Scale (25\%). The most prevalent diagnosis was mild TBI (25\%), followed by post-traumatic stress disorder (PTSD) (17.5\%), and a subgroup within our epilepsy sample consisting of a combination of subjects with a diagnosis of epilepsy, or mixed seizure disorder (17.5\%).

A large number of forensic participants $(32 \%)$ had a primary diagnosis of mild TBI, and it is reasonable to assume that these are the ones more likely to be told about cognitive rest, etc. (perhaps creating a cogniphobic response) versus epilepsy participants, etc., who never get this message. Future studies are needed to investigate the role of specific diagnoses, for example, mild TBI and epilepsy, and diagnosis threat in the genesis of Cogniphobia. Our results indicate that Cogniphobia is an important psychological construct that appears to be related to PVT failure, and occurs more frequently at elevated levels in a forensic sample.

The psychological construct Symptom Identity was also a significant predictor of PVT failure. As scores increased 
for Symptom Identity, the risk of PVT failure also increased. However, Symptom Identity scores exhibited a "threshold" effect with scores $\geq 24$ associated with more than an 11-fold increase in the odds of PVT failure compared to examinees with a score $<24$. Twenty-seven of 167 participants $(16.2 \%)$ scored $\geq 24$ on the Symptom Identity Scale. Twenty-four of the 27 high scorers $(88.8 \%)$ were examined in a forensic context, which was significantly greater than the percentage of clinical nonforensic participants scoring $\geq 24$ on the Symptom Identity Scale $(25 \%)$. The most prevalent diagnosis was mild TBI (28\%), followed by PTSD and moderate-severe TBI (20\%, respectively). The present study has expanded evidence for a relationship between Symptom Identity and PVT performance, although once again, as with Cogniphobia, extremely elevated scores occurred at a higher percentage in participants examined in a forensic context.

The finding that illness perceptions are significant predictors of cognitive performance validity in a mixed forensic and nonforensic clinical sample suggests that performance invalidity is more complex than historically indicated by its relationship with the MMPI-2-RF validity scales. The present finding requires cross validation before routine clinical use would be considered, and in the meantime clinicians should continue to consider the MMPI-2-RF validity scales in all of their clinical assessments. The current findings may prompt some clinicians to reconsider the influence of other factors when interpreting PVT performance.

\section{Limitations and Suggestions}

First, although the current study represents a fairly large sample size for neuropsychological research, it has to be viewed as an exploratory study and relatively small in scale for the statistical analyses used. Thus, further replication in a larger independent sample will be necessary. Second, given that $44 \%$ of our participants were referred by attorneys or disability carriers, we cannot rule out the potential for referral bias as participants were not randomly selected. However, results were in the expected direction as $39 \%$ of our sample produced noncredible PVT performance. Third, while backward (and forward) stepwise variable selection techniques have a high likelihood of capitalizing on chance, a gradient boost regression selection, as well as a logistic regression blocking procedure, identified the same three predictors out of the pool of 14 potential predictors providing some reassurance that the results are not statistical artifacts. Moreover, variables identified by stepwise search are less likely to be artifacts when correlations among them are mostly low to moderate as shown in Table 6.

Fourth, the current results do not extend to a criminal context. Fifth, the Symptom Identity and Cogniphobia constructs that emerged in the current exploratory prospective study may function as "psychological proxies" for cognitive performance validity in situations where traditional stand alone and embedded performance validity measures are absent or lacking. This simply means that in a database devoid of any cognitive PVTs, but where there are scores for Cogniphobia and/or Symptom Identity, then scores on the latter instruments may serve as "proxies", that is, substitutes, to provide some guidance on credibility of neuropsychological performance. High scores suggest a relationship between abnormal health beliefs and cognitive performance validity. While the confidence bounds for the ORs for Symptom Identity are wide, the more critical clinically relevant statistics for predicting PVT credibility are not the odds ratios, but the sensitivity, specificity, and accuracy. The 74\% accuracy in our study suggests that there are other factors that affect PVT credibility and ideally these results should be validated in a future study with a larger sample size.

Sixth, while Cogniphobia and Symptom Identity were the best predictors of PVT performance, it is important to remember that these two illness perceptions can co-exist with malingering. Clinical judgment pertaining to their co-existence, or lack thereof, needs to be based upon consideration of the entire neuropsychological database including not only context of the examination, but also clinical history and evolution of symptom presentation. As extreme elevations on the relevant intrinsic psychological variables were more common in the forensic setting, it would be useful to explore these findings in a sample with significant somatization tendencies who lack external incentive [e.g., pseudoneurologic epileptic seizures (PNES) patients with no evidence of financial incentive]. This would add confidence to the suggestion that PVT measures can be failed due to variables other than intentional underperformance.

Of note, a compelling case for this possibility has been made in the PNES population, where researchers demonstrated that financial incentive was not a contributing factor to PVT performance (Williamson, et al., 2005). Future studies with a PNES or similar somatic/functional population with or without external incentive would be useful for further confirmation and clarification of these findings.

Finally, on a somewhat cautionary note, for clinicians engaged in the assessment of patients with epilepsy, the current findings highlight the possible effect of interictal epileptiform discharges on cognitive performance and PVT measures. One of the clinical samples included a large number of epilepsy patients, and all of these patients had simultaneous EEG data during their test sessions. We excluded any patients with recent $(<24 \mathrm{hr})$ or concurrent epileptiform activity based on recent studies suggesting a relationship between these variables (Drane et al., 2016; Williamson et al., 2005). An elevated rate of PVT failure was noted in our epilepsy sample with concurrent epileptiform activity, which suggests a possible relationship between verbal PVTs and dominant TL epileptiform activity. This should represent a caution to studies involving patients with epilepsy, as well as clinical neuropsychological assessment of this patient population as well. Future studies are needed to further explore the relationship between dominant left temporal lobe epileptiform activity and performance on verbal PVTs. 


\section{CONCLUSION}

Current findings implicate psychological factors in the form of illness perceptions that need to be considered when analyzing PVT performance (i.e., not limited to malingering) and can co-exist with malingering. Psychological correlates of cognitive performance validity deserve additional investigation and consideration when interpreting patient profiles generated during forensic and clinical neuropsychological examinations.

\section{ACKNOWLEDGMENTS}

Authors (G.H., R.H.) declare there was a fee for service for the 81 forensic referrals, while authors (J.S., J.G., W.E., D.D.) report no potential conflicts of interest. Partial funding was provided by NCS Pearson, Minneapolis, MN, for MMPI-2-RF scoring costs.

\section{REFERENCES}

American Congress of Rehabilitation Medicine. (1993). Definition of mild traumatic brain injury. Journal of Head Trauma Rehabilitation, 8, 86-87.

Amrhein, C., Hengmith, S., Maragkos, M., \& Hennig-Fast, K. (2008). Neuropsychological characteristics of highly dissociative healthy individuals. Journal of Trauma Dissociation, 9, 525-542.

An, K.Y., Zakzanis, K.K., \& Joordens, S. (2012). Conducting research with nonclinical healthy undergraduates: Does effort play a role in neuropsychological performance? Archives of Clinical Neuropsychology, 27, 849-857.

Ardolf, B.K., Denney, R.L., \& Houston, C.M. (2007). Base rates of negative response bias and malingered neurocognitive dysfunction among criminal defendants referred for neuropsychological evaluation. The Clinical Neuropsychologist, 21, 899-916.

Bandura, A. (1997). Self-efficacy: The exercise of control. New York: Freeman.

Barash, J., Suhr, J.A., \& Manzel, K. (2004). Detecting poor effort and malingering with an expanded version of the Auditory Verbal Learning Test (AVLT): Validation with clinical samples. Journal of Clinical and Experimental Neuropsychology, 26, 125-140.

Belanger, H.G., Barwick, F.H., Kip, K.E., Kretzmer, T., \& Vanderploeg, R.D. (2013). Postconcussive symptom complaints and potentially malleable positive predictors. The Clinical Neuropsychologist, 27, 343-355.

Ben-Porath, Y.S., \& Tellegen, A. (2008). Minnesota Multiphasic Personality Inventory-2-Restructured Form: Manual for administration, scoring, and interpretation. Minneapolis, MN: University of Minnesota by NCS Pearson, Inc.

Bernstein, E.M., \& Putnam, F.W. (1986). Development, reliability, and validity of a dissociation scale. Journal of Nervous and Mental Diseases, 174, 727-734.

Boone, K.B., Salazar, X., Lu, P., Warner-Chacon, K., \& Razani, J. (2002). The Rey 15-item recognition trial: A technique to enhance sensitivity of the Rey 15-item memorization test. Journal of Experimental Neuropsychology, 24, 561-573.

Boone, K., Lu, P., \& Wen, J. (2005). Comparison of various RAVLT scores in the detection of noncredible memory performance. Archives of Clinical Neuropsychology, 20, 301-319.

Crombez, G., Verbaet, L., Lysens, R., Baeyers, F., \& Eelen, P. (1998). Avoidance and confrontation of painful, back straining movements in chronic back pain patients. Behavior Modification, $22,62-77$.
Dean, A.C., Victor, T.L., Boone, K.B., \& Arnold, G. (2008). The relationship of IQ to effort test performance. The Clinical Neuropsychologist, 22, 705-722.

Dean, A.C., Victor, T.L., Boone, K.B., Philpott, L., \& Hess, R. (2009). Dementia and effort test performance. The Clinical Neuropsychologist, 23, 133-152.

Delis, D.C., Kramer, J.H., Kaplan, E., \& Ober, B.A. (2000). California Verbal Learning Test-II (2nd Ed.). San Antonio, TX: The Psychological Corporation.

Delis, D.C., \& Wetter, S.R. (2007). Cogniform Disorder and Cogniform Condition: Proposed diagnoses for excessive cognitive symptoms. Archives of Clinical Neuropsychology, 22, 589-604.

DePrince, A.P., \& Freyd, J.J. (1999). Dissociative tendencies, attention and memory. Psychological Science, 10, 449-452.

DeRight, J., \& Jorgensen, R.S. (2015). I just want my research credit: Frequency of suboptimal effort in a non-clinical healthy undergraduate sample. The Clinical Neuropsychologist, 29, 101-107.

Diefenbach, M.A., \& Leventhal, H. (1996). The Common-Sense Model of Illness Representation: Theoretical and practical considerations. Journal of Social Distress and the Homeless, 5, $11-38$.

Drane, D.L., Williamson, D.J., Stroup, E.S., Holmes, M.D., Jung, M., Koerner, E., \& Miller, J.W. (2006). Cognitive impairment is not equal in patients with epileptic and psychogenic nonepileptic seizures. Epilepsia, 47, 1879-1886.

Drane, D.L., Ojemann, J.G., Kim, M., Gross, R.E., Miller, J.W., Faught, R.E. Jr., \& Loring, D.W. (2016). Interictal epileptiform discharge effects on neuropsychological assessment and epilepsy surgical planning. Epilepsia and Behavior, 56, 131-138.

Forbey, J.D., \& Lee, T.T.C. (2011). An exploration of the impact of invalid MMPI-2 protocols on collateral self-report measure scores. Journal of Personality Assessment, 93, 556-565.

Forbey, J.D., Lee, T.C.C., Ben-Porath, Y.S., Arbisi, P.A., \& Gartland, D. (2013). Associations between MMPI-2-RF validity scales and extra-test measure of personality and psychopathology. Assessment, 20, 448-461.

Fragale, A.R., \& Health, C. (2004). Evolving informational credentials: The (mis) attribution of believable facts to credible sources. Personality and Social psychology Bulletin, 30, 226-236.

Freyd, J., Martorello, S.R., Alvarado, J.S., Hayes, A.E., \& Christman, J.C. (1998). Cognitive environments and dissociative tendencies: Performance on the Standard Stroop Task for high versus low dissociators. Applied Cognitive Psychology, 12, S91-S103.

Gervais, R.O., Ben-Porath, Y.S., Wygant, D.B., \& Green, P. (2007). Development and validation of a Response Bias Scale (RBS) for the MMPI-2. Assessment, 14, 196-208.

Gervais, R.O., Wygant, D.B., Sellbom, M., \& Ben-Porath, Y.S. (2011). Associations between Symptom Validity Test failure and scores on the MMPI-2-RF validity and substantive scales. Journal of Personality Assessment, 93, 508-517.

Green, P. (2005). Green's Word Memory Test for Windows: User's manual. Edmonton, Canada: Green's Publishing, Inc.

Greiffenstein, M., Baker, W., \& Gola, T. (1994). Validation of malingered amnesia measures with a large clinical sample. Psychological Assessment, 6, 218-224.

Greve, K.W., Heinly, M.T., Bianchini, K.J., \& Love, J.M. (2009). Malingering detection with the Wisconsin Card Sorting Test in mild traumatic brain injury. The Clinical Neuropsychologist, 23, $343-362$ 
Haggerty, K.A., Frazier, T.W., Busch, R.M., \& Naugle, R.I. (2007). Relationships among Victoria Symptom Validity Test indices and personality assessment inventory validity scales in a large clinical sample. The Clinical Neuropsychologist, 21, 917-928.

Henry, G.K., Heilbronner, R.L., Algina, J., \& Kaya, Y. (2013). Derivation of the MMPI-2-RF Henry-Heilbronner Index-r (HHI-r) Scale. The Clinical Neuropsychologist, 27, 509-515.

Ingram, P.B., \& Ternes, M.S. (2016). The detection of contentbased invalid responding: A meta-analysis of the MMPI-2-RF over-reporting scales. The Clinical Neuropsychologist, 30, 473-496.

Jones, A., Ingram, V.M., \& Ben-Porath, Y.S. (2012). Scores on the MMPI-2-RF Scales as a function of increasing levels of failure on cognitive symptom validity tests in a military sample. The Clinical Neuropsychologist, 26, 790-815.

Johnson, S.K. (2008). Medically unexplained illness: Gender and biopsychosocial implications. Washington, DC: American Psychological Association.

Kemp, S., Coughlan, A.K., Rowbottom, C., Wilkinson, K., Teggart, V., $\&$ Baker, G. (2009). The base rate of effort test failure in patients with medically unexplained symptoms. Journal of Psychosomatic Research, 65, 319-325.

Kotor, R.I., Bellman, S.B., \& Watson, D.B. (2004). Multidimensional Iowa Suggestibility Scale. Stony Brook University: roman. Kotov@stonybrook.edu.

Lamberty, G.J. (2008). Understanding somatization in the practice of clinical neuropsychology. New York: Oxford University Press, Inc.

Larrabee, G.J. (2003). Detection of malingering using atypical performance patterns on standard neuropsychological tests. The Clinical Neuropsychologist, 17, 410-425.

Lees-Haley, P.R., Iverson, G.L., Lange, R.T., Fox, D.D., \& Allen, L.M., III (2002). Malingering in forensic neuropsychology: Daubert and the MMPI-2. Journal of Forensic Neuropsychology, $3,167-203$.

Loring, D.W., Lee, G.P., \& Meador, K.J. (2005). Victoria Symptom Validity Test performance in nonlitigating epilepsy surgery candidates. Journal of Clinical and Experimental Neuropsychology, 27, 610-617.

Martelli, M.F., Zasler, N.D., Grayson, R.I., \& Liljedahl, E.L. (1999). Kinesiophobia and cogniphobia: Assessment of avoidance conditioned pain related disability (ACPRD). Poster presentation. National Academy of Neuropsychology, San Antonio, TX.

Martin, P.K., Schroeder, R.W., Heinrichs, R.J., \& Baade, L.E. (2015). Does true neurocognitive dysfunction contribute to Minnesota Multiphasic Personality Inventory-2 Edition-Restructured Form cognitive validity scale scores? Archives of Clinical Neuropsychology, 30, 377-386.

Martin, P.K., Schroeder, R.W., \& Odland, A.P. (2015). Neuropsychologists' validity testing beliefs and practices: A survey of North American professionals. The Clinical Neuropsychologist, 29, 741-776.

Martin, P.K., Schroeder, R.W., \& Odland, A.P. (2016). Expert beliefs and practices regarding neuropsychological validity testing. The Clinical Neuropsychologist, 30, 515-535.

Mittenberg, W., DiGiulio, V., Perrin, S., \& Bass, A.E. (1992). Symptoms following mild head injury: Expectation as aetiology. Journal of Neurology, Neurosurgery, and Psychiatry, 55, 200-204.

Mittenberg, W., Tremont, G., Zielinski, R.E., Fichera, S., \& Rayls, K.R. (1996). Cognitive behavioral prevention of postconcussion syndrome. Archives of Clinical Neuropsychology, 11, 139-145.
Mittenberg, W., Patton, C., Canyock, E.M., \& Condit, D.C. (2002). Base rate of malingering and symptom exaggeration. Journal of Clinical and Experimental Neuropsychology, 24, 1094-1102.

Moss-Morris, R., Weinman, J., Petrie, K.J., Horne, R., Cameron, L.D., \& Buick, D. (2002). The Revised Illness Perception Questionnaire (IPQ-R). Psychology and Health, 17, 1-16.

Nelson, N.W., Hoelzle, J.B., McGuire, K.A., Sim, A.H., Goldman, D., Ferrier-Auerbach, A.G., ... Sponheim, S.R. (2011). Self-report of psychological function among OEF/OIF personnel who also report combat-related concussion. The Clinical Neuropsychologist, 25, 716-740.

Nguyen, C.T., Green, D., \& Barr, W.B. (2015). Evaluation of the MMPI-2-RF for detecting over-reported symptoms in a civil forensic and disability setting. The Clinical Neuropsychologist, 2 , 255-271.

Peck, C.P., Schroeder, R.W., Heinrichs, R.J., VonDran, E.J., Brockman, C.J., Webster, B.K., \& Baade, L.E. (2013). Differences in MMPI-2 FBS and RBS scores in brain injury, probable malingering, and conversion disorder groups: A preliminary study. The Clinical Neuropsychologist, 27, 693-707.

Rogers, R.R., Gillard, N.D., Berry, D.T.R., \& Granacher, R.P. (2011). Effectiveness of the MMPI-2-RF validity scales for feigned mental disorders and cognitive impairment. Journal of Psychopathology and Behavioral Assessment, 33, 355-367.

Schroeder, R.W., Baade, L.E., Peck, C.P., VonDran, E.J., Brockman, C.J., Webster, B.K., \& Heinrichs, R.J. (2012). Validation of MMPI-2-RF validity scales in criterion group neuropsychological samples. The Clinical Neuropsychologist, 26, 129-146.

Schroeder, R.W., \& Marshall, P.S. (2011). Evaluation of the appropriateness of multiple symptom validity indices in psychotic and non-psychotic psychiatric populations. The Clinical Neuropsychologist, 25, 437-453.

Schwarzer, R., \& Jerusalem, M. (1995). The General Self-Efficacy Scale. In J. Weinman, S. Wright, \& M. Johnson (Eds.), Measures in health psychology: A user's portfolio. Casual and central beliefs. Windsor, UK: NFER-NELSON.

Sellbom, M., \& Bagby, R.M. (2010). Detection of over reported psychopathology with the MMPI-2-RF [corrected] validity scales. Psychological Assessment, 22, 757-767.

Sellbom, M., Toomey, J.A., Wygant, D.B., Kucharski, L.T., \& Duncan, S. (2010). Utility of the MMPI-2-RF (restructured form) validity scales in detecting malingering in a criminal forensic setting: a known-groups design. Psychological Assessment, 22, 22-31.

Silk-Eglit, G.M., Stenclik, J.H., Gavett, B.E., Adam, J.W., Lynch, J.K., \& McCaffrey, R.J. (2014). Base rate of performance invalidity among non-clinical undergraduate research participants. Archives of Clinical Neuropsychology, 29, 415-421.

Slick, D., Hopp, M.A., Strauss, E., \& Thompson, G.B. (1997). Victoria Symptom Validity Test. Lutz, FL: Psychological Assessment Resources.

Snell, D.L., Hay-Smith, E.J., Surgenor, L.J., \& Siegert, R.J. (2013). Examination of outcome after mild traumatic brain injury: The contribution of injury beliefs and Leventhal's common sense model. Neuropsychological Rehabilitation, 23, 333-362.

Suhr, J.A. (2003). Neuropsychological impairment in fibromyalgia: Relation to depression, fatigue and pain. Journal of Psychosomatic Research, 55, 321-329.

Suhr, J.A., \& Spickard, B. (2012). Pain-related fear is associated with cognitive task avoidance: Exploration of the cogniphobia construct in a recurrent headache sample. The Clinical Neuropsychologist, 26, 1128-1141. 
Sweet, J.J., Condit, D.C., \& Nelson, N.W. (2008). Feigned amnesia and memory loss. In R. Rogers (Ed.), Clinical assessment of malingering and deception (3rd ed.). New York: Guilford Press.

Tarescavage, A.M., Wygant, D.B., Gervais, R.O., \& Ben-Porath, Y.S. (2012). Association between the MMPI-2 restructured form (MMPI-2-RF) and malingered neurocognitive dysfunction among non-head injury disability claimants. The Clinical Neuropsychologist, 27, 313-335.

Thomas, M.L., \& Youngjohn, J.R. (2009). Let's not get hysterical: Comparing the MMPI-2 validity, clinical, and RC scales in TBI litigants tested for effort. The Clinical Neuropsychologist, 23, 1067-1084.

Todd, D.D., Martelli, M.,.F., \& Grayson, R.L. (1998). The Cogniphobia Scale (C-Scale) (white paper). Retrieved from www.angelfire.com/va/MFMartelliPhD/nanposters.html.

Tombaugh, T.N. (1996). Test of Memory Malingering. North Tonawanda, NY: Multi-Health Systems, Inc.

Tutz, G., \& Binder, H. (2006). Generalized additive modeling with implicit variable selection by likelihood-based boosting. Biometrics, 62, 961-971.

Van Dyke, S.A., Millis, S.R., Axelrod, B.N., \& Hanks, R.A. (2013). Assessing effort: Differentiating performance and symptom validity. The Clinical Neuropsychologist, 27, 1234-1246.

Vaquez-Justo, E., Alvarez, M.R., \& Otero, M.J.F. (2003). Influence of depressed mood on neuropsychological performance in HIVseropositive drug users. Psychiatry and Clinical Neurosciences, 57, 251-258.

Whittaker, R., Kemp, S., \& House, A. (2007). Illness perceptions and outcome in mild head injury: A longitudinal study. Journal of Neurology, Neurosurgery, and Psychiatry, 78, 644-646.
Williamson, D.J., Drane, D.L., Stroup, E.S., Holmes, M.D., Wilensky, A.J., \& Miller, J.W. (2005). Recent seizures may distort the validity of neurocognitive test scores in patients with epilepsy. Epilepsia, 46(Suppl. 8), 74.

Williamson, D.J., Holsman, M., Clayton, N., Miller, J.W., \& Drane, D. (2012). Abuse, not financial incentive predicts noncredible cognitive performance in patients with psychogenic nonepileptic seizures. The Clinical Neuropsychologist, 26, 588-598.

Wolfe, P.L., Millis, S.R., Hanks, R., Fichtenber, N., Larrabee, G.J., \& Sweet, J.J. (2010). Effort indicators within the California Verbal Learning Test-II (CVLT-II). The Clinical Neuropsychologist, 24, 153-168.

Wygant, D.B., Sellbom, M., Gervais, R.O., Ben-Porath, Y.S., Stafford, K.P., Freeman, D.B., \& Heilbronner, R.L. (2010). Further validation of the MMPI-2 and MMPI2-RF Response Bias Scale: Findings from disability and criminal forensic settings. Psychological Assessment, 22, $745-756$

Wygant, D.B., Ben-Porath, Y.S., Arbisi, P.A., Berry, D.T., Freeman, D.B., \& Heilbronner, R.L. (2009). Examination of the MMPI-2 restructured form (MMPI-2-RF) validity scales in civil forensic settings: Findings from simulation and known group samples. Archives of Clinical Neuropsychology, 24, 671-680.

Youngjohn, J.R., Wershba, R., Stevenson, M., Sturgeon, J., \& Thomas, M.L. (2011). Independent validation of the MMPI-2-RF somatic/cognitive and validity scales in TBI litigants tested for effort. The Clinical Neuropsychologist, 25, 463-476. 